\title{
MERCOSUL E O EFEITO SPILLOVER NA CRIAÇÃO DE COMÉRCIO*
}

\author{
Alexandre Loures ${ }^{\dagger}$ \\ ERIK Figueiredo ${ }^{\ddagger}$ \\ LUCAS MARIANO $§$
}

\begin{abstract}
Resumo
Este artigo analisa se a experiência de exportação dentro do Mercosul afeta a probabilidade de criação de mercado para novos produtos fora da área de comércio, i.e., um efeito spillover para terceiros países. Usando um banco de dados com elevada desagregação (BACI-HS4) e uma estratégia de estimação baseada em modelos probabilísticos, constata-se que as experiências anteriores de exportação estão associadas a uma probabilidade de 1,9\% de criação de mercado para novos produtos. Esse efeito, aliado aos resultados de Figueiredo \& Loures (2016), indicam que o Mercosul não só contribui para a ampliação da margem extensiva de comércio dentro do bloco, como também constitui um primeiro passo para que um produto se consolide na exploração de novos mercados internacionais.
\end{abstract}

Palavras-chave: Mercosul, comércio internacional, efeito spillover.

\begin{abstract}
This paper analyzes whether export experience within Mercosur affects the likelihood of market creation for new non-marketed products, i.e. a spillover effect for third countries. Using a highly disaggregated database (BACI-HS4) and an estimation strategy based on probabilistic models, it is found that previous export experiences are associated with a $1.9 \%$ market-creation probability for new products. This effect, coupled with the results of Figueiredo \& Loures (2016), indicates that Mercosur not only contributes to the expansion of the extensive trade margin within the bloc, but also constitutes a first step for a product to consolidate itself in the exploration of new international markets.
\end{abstract}

Keywords: Mercosur, international trade, spillover effect.

JEL classification: C33, C55, F14, F15.

DOI: http://dx.doi.org/10.11606/1980-5330/ea150797

\footnotetext{
* Os autores são gratos ao suporte do CNPq por meio do projeto 400216/2017-5.

† Universidade Federal da Paraíba (UFPB), Brasil. Email: alexandre.loures@ymail.com

‡ Universidade Federal da Paraíba (UFPB), Brasil. Email: eafigueiredo@gmail.com

$\S$ Universidade Federal da Paraíba (UFPB), Brasil. Email: lucasmariano1905@gmail.com
} 


\section{Introdução}

Na segunda metade da década de 2000, o Inter-American Development Bank promoveu uma série de discussões relacionadas ao futuro do Mercosul. O esforço dos diversos participantes resultou em uma coletânea de artigos sintetizadas no livro "Deepening Integration in Mercosur: Dealing with Disparities" organizado por Blyde et al. (2008). O tema central do debate era como um acordo regional poderia sobreviver a disparidades institucionais e de política macroeconômica apresentadas por seus países fundadores. Em meio a tantas dúvidas, alguns pesquisadores mostravam-se otimistas quanto ao sucesso da integração desses países. Galvão Jr (2008, p. 255), por exemplo, classificava o Mercosul como sendo um "young and highly asymmetric integration effort" $\mathrm{e}$, como um acordo 'jovem', ele teria "time to solve or at least accommodate, within the integration framework, most of its numerous problems".

Em oposição às previsões otimistas, estudos como os de Olarreaga \& Soloaga (1998), Azevedo (2008) e Reis et al. (2014) alertavam para os possíveis desvios de comércio gerados pelo bloco. ${ }^{1}$ Em outras palavras, dividindo o comércio em duas partes, a intra-bloco - entre os países signatários - e a extrabloco - entre os signatários e parceiros fora do Mercosul -, observou-se que as exportações extra-bloco foram reduzidas devido à preferência dada em exportar para dentro do bloco, o que colocava em dúvida o efeito líquido da criação de novas exportações.

Hoje, quase trinta anos depois de sua fundação, pode-se dizer que as previsões otimistas, como as de Galvão Jr (2008), têm prevalecido. De uma forma geral, as evidências empíricas têm indicado diversos benefícios econômicos do Mercosul sobre os seus países membros. Os exemplos são diversos, Yeats (1997), pioneiramente, indentificou um crescimento expressivo no comércio intra-bloco principalmente em produtos nos quais o bloco não era competitivo internacionalmente. Cordeiro \& Rodrigues Jr (2016) chegam a conclusões similares, porém, apontando indícios de desvio de exportações industriais em direção do bloco ("trade diversion"). Usando uma abordagem estrutural, Loures \& Figueiredo (2017) sugerem que o acordo gera um impacto positivo sobre o bem-estar econômico dos países membros. Os mesmos autores, Figueiredo \& Loures (2016), também demonstram que o bloco promove a entrada de novos produtos no mercado, comprovando seu efeito sobre a margem extensiva de comércio. ${ }^{2}$

Diante disso, este artigo pretende testar uma hipótese ainda pouco explorada pela literatura relacionada aos efeitos dos "Regional Trade Agreements" (RTAs), qual seja: que esses acordos são capazes de gerar externalidades positivas na pauta de exportação (efeitos de spillover). Mais especificamente, buscase averiguar se o Mercosul não só promove a entrada de novos produtos no setor exportador, como constitui o primeiro passo para que esses produtos sejam ofertados no mercado internacional posteriormente.

\footnotetext{
${ }^{1}$ Há também discussões relacionadas aos problemas de formação/consolidação do bloco. Preusse (2001, p. 21), por exemplo, destaca as tensões existentes: “ (...) some important obstacles have to be removed: first, there are strong vested interests in the leading economies of the region which try heavily to avoid rigorous structural adjustment to the world market. This adjustment, however, is indispensable if Mercosur is to become an open region after a long period of import substitution".

${ }^{2}$ Margem extensiva caracteriza-se como sendo o número de produtos comercializados, enquanto margem intensiva é a média do valor dos produtos já comercializados no mercado internacional (Baldwin \& Di Nino 2008, Santos Silva et al. 2014).
} 
Essa hipótese está ancorada em desenvolvimentos teóricos recentes e seus mecanismos causais podem ser classificados em três efeitos distintos: i) a existência de economias de escala na produção, ${ }^{3}$ ii) a presença de experiência adquirida nas exportações para países membros do acordo ${ }^{4}$ - "learning by exporting" - e, iii) a ocorrência de economias de escala na exportação para países não-membros do RTA. ${ }^{5}$ Conforme Krugman (1980), quando um acordo é formado, as firmas conseguem distribuir os custos para um mercado maior, ou seja, para um maior número de consumidores, o que acaba favorecendo o aumento do comércio considerando uma situação em que as firmas têm economias de escala na produção e, assim, aumentando a posssibilidade de exportar para terceiros países. Ademais, após a promulgação de um RTA, as firmas ficam expostas a um maior mercado exportador, o que poderia aumentar sua produtividade, reduzindo seus custos e permitindo que elas consigam exportar para mercados fora do bloco cujos custos transacionais inviabilizavam os fluxos bilaterais de comércio anteriormente.

Para este artigo, define-se um novo produto, $k$, como sendo aquele produto para o qual não houve exportação em qualquer um dos anos anteriores à promulgação do Mercosul (1991). Em seguida, verifica-se se esse novo produto foi comercializado primeiramente entre os membros do Mercosul para, só depois, fazer parte da pauta exportadora dos membros e não-membros. Em caso afirmativo, tem-se uma evidência preliminar de que o Mercosul age como um primeiro passo para a consolidação de um produto no mercado internacional.

Para fundamentar econometricamente a hipótese do efeito spillover, este estudo adotará uma estratégia similar à de Molina (2010). Nesse sentido, buscase analisar o impacto do acordo de livre comércio para os países do Mercosul sobre a probabilidade das exportações de novos produtos para terceiros países. Será considerada uma série de especificações econométricas visando controlar por fatores não-observados e correlacionados com a variável de interesse. Os resultados inciais serão baseados em um modelo binário, logit, sem inclusão de efeitos fixos, dado o conhecido problema de "parâmetros incidentais" associados a essa classe de estimadores (Neyman \& Scott 1948). Modelos com efeitos fixos e variantes no tempo serão abordados a partir de uma estrutura de probabilidade linear, estimada como uma análise de robustez.

A organização deste artigo tem a seguinte forma. Além dessa seção, encontrase apresentada na Seção 2 a especificação econométrica utilizada para as análises. Já na Seção 3 apresentam-se os resultados e discussões. Por fim, na Seção 4 são feitas as considerações finais.

\footnotetext{
${ }^{3}$ Conforme Moore (1959) economia de escala caracteriza-se como sendo a vantagem de custo que a empresa obtém devido sua escala de operação em que o custo por unidade de produto diminui com o aumento da escala.

${ }^{4}$ Para Molina (2010) a exposição das firmas aos mercados internacionais pode contribuir para que possam ter acesso a novas tecnologias e expertises, contribuindo para melhorar seu processo de produção e, assim, reduzindo seus custos de produção.

${ }^{5}$ Destaca-se a existência de um custo de entrada no mercado internacional associado com o estabelecimento de canais de distribuição, mas uma vez estabelecidos esses canais, o custo médio de exportar o mesmo produto para destinos adicionais deve ser menor (Borchert 2007).
} 


\section{Dados e especificação empírica}

\subsection{Dados}

Fluxo de comércio bilateral: a principal fonte de dados é constituída por informações relativas ao comércio bilateral positivo (ausência de zeros) entre os países de origem e destino, respectivamente, $i$ e $j$. Considerou-se a desagregação do Sistema Harmonizado de quatro dígitos (HS4), que abrange o maior número de países possível, totalizando, em média, 1.241 setores para cada país. Os dados são fornecidos pelo Centre D'Estudes Prospectives Et D'Informations Internationales (CEPII), Base pour l'Analyse du Commerce International (BACI); ${ }^{6}$

Regional Trade Agreements - RTAs: os dados sobre os RTAs foram obtidas da base Mario Larch's Trade Agreements Database; ${ }^{7}$

Demais variáveis de controle: outra fonte de informação foi a The Database Penn World Table version 9.0, de onde foram obtidos dados sobre o Produto Interno Bruto (PIB), taxa de câmbio real e sobre o PIB per capita; ${ }^{8}$

Período de análise e definição do Mercosul: a análise compreende o período amostral 1989-2010, sendo a amostra composta pelos membros plenos do Mercosul: Argentina, Brasil, Paraguai e Uruguai como países de origem e 183 países como destino, ${ }^{9}$ totalizando 338.822 observações. Destaca-se que, uma vez que a Venezuela atualmente encontra-se suspensa do acordo bem como se tornou membro pleno do Mercosul ${ }^{10}$ somente em 2012 e o período amostral termina em 2010, este artigo optou por não incluir essa economia como um país de origem na análise e;

Fluxos comerciais iguais a zero: convém ressaltar que a base BACI, utilizada neste estudo, não reporta a ausência de comércio, ou seja, o fluxo de comércio bilateral igual a zero. Dessa forma, o presente estudo realizou um procedimento de expansão da base de tal modo que esse fluxo fosse computado na base.

\subsection{Definições: novos produtos e spillover}

Considere a identificação de um novo produto, $k$, na pauta de exportação do país $i$, no tempo $t$. Assuma ainda que ele não era exportado por $i$ no período $t-n$. Algebricamente, tal situação pode ser representada da seguinte forma:

$$
N e w P_{i k, t}= \begin{cases}1 & \text { se } x_{i k, t}>0 \text { e } x_{i k, t^{*}}=0 \text { para } t^{*}<t \\ 0 & \text { caso contrário }\end{cases}
$$

em que $x_{i k, t}$ representa o valor exportado do produto $k$ do país de origem $i$ no período $t$.

\footnotetext{
${ }^{6}$ Disponível em: http://www.cepii.fr/CEPII/en/bdd_modele/presentation asp? $\mathrm{id}=1$.

${ }^{7}$ Obtida no endereço: http: //www. ewf .uni-bayreuth.de/en/research/RTA-data/ index.html.

${ }^{8}$ Essa base encontra-se em: https: / /www.rug.nl/ggdc/productivity/pwt/.

${ }^{9} \mathrm{~A}$ lista de todos os países de destino encontra-se na Tabela 3 do Apêndice A-1.

${ }^{10}$ O Mercosul é composto por cinco membros plenos (Argentina, Brasil, Paraguai, Uruguai e Venezuela - atualmente suspensa), cinco membros associados (Bolívia, Chile, Colômbia, Equador e Peru) e dois estados observadores (Nova Zelândia e México).
} 
Conforme Molina (2010), essa delimitação para novos produtos se faz necessária para identificar onde a experiência pregressa da exportação ocorreu. Sendo assim, a fim de determinar se a experiência foi adquirida no comércio intra-bloco ou fora do bloco, extraem-se dois subconjuntos do conjunto dado na Condição 1. A seguir, tem-se o subconjunto para as experiências adquiridas através do fluxo bilateral de comércio com os países não-membros, representados por $j$ :

$$
N e w P_{i j k, t}= \begin{cases}1 & \text { se } x_{i j k, t}>0 \text { e } x_{i j k, t^{*}}=0 \text { para } t^{*}<t \\ 0 & \text { caso contrário }\end{cases}
$$

Por sua vez, o subconjunto para as experiências adquiridas por meio do comércio entre os membros do Mercosul, representados por $m$, é dado por:

$$
N e w P_{i m k, t}= \begin{cases}1 & \text { se } x_{i m k, t}>0 \text { e } x_{i m k, t^{*}}=0 \text { para } t^{*}<t \\ 0 & \text { caso contrário }\end{cases}
$$

Diante disso, no contexto do presente estudo, define-se a variável que irá capturar o efeito das experiências anteriores entre os membros do Mercosul por:

$$
\text { inside }_{i j k, t}= \begin{cases}1 & \text { se } x_{i j k, t}>0 \quad \forall j \text { e } t \in[t-2 ; t-1] \\ 0 & \text { caso contrário }\end{cases}
$$

Importante salientar que a construção para a variável que irá capturar as experiências anteriores entre membros e não-membros, outside $e_{i j k, t}$, é idêntica a da inside $e_{i j k, t}$ e, por brevidade, não será mostrada aqui. Ademais, a variável que irá capturar o efeito spillover caracteriza-se como sendo uma interação entre uma dummy que assume valor igual a um se o par de países $i$, origem, e $j$, destino, são membros do Mercosul e zero caso contrário, capturando o efeito do Mercosul - denotada por mercosur $r_{i j, t}$ - e a variável explicativa inside $e_{i j k, t}$.

Definidas as variáveis centrais do estudo, a subseção seguinte discute a especificação econométrica adotada para a mensuração do efeito spillover.

\subsection{Especificação empírica}

Como já ressaltado, a base de dados utilizada para a construção da variável dependente deste estudo não reporta os fluxos de comércio bilateral iguais a zero. Portanto, o primeiro passo será o da expansão da base BACI, de modo a reportar os setores inexistentes na pauta de exportação com valores iguais a zero. Por exemplo, suponha que o Brasil não tenha exportado soja para os Estados Unidos nos anos de 2000 e 2001, o que não será reportado na BACI, mas ao expandir essa base, essa ausência de comércio na commodity soja entre Brasil e Estados Unidos será gerada e reportada com valor zero. Em seguida, cria-se uma variável indicadora que assume valor 1 , se o valor exportado do setor é positivo e zero caso contrário.

Para identificar o fluxo de novos produtos nos diferentes mercados, serão necessárias duas definições prévias relativas aos novos produtos e os grupos 
de destino desses produtos. Em primeiro lugar, um novo produto será aquele que nunca fez parte da pauta de exportação dos países exportadores no período antes da promulgação do Mercosul. Esse novo produto poderá ser destinado a dois grupos distintos de importadores: a) os países membros do Mercosul e; b) para os países não-membros do Mercosul (por vezes chamados de terceiros países). Dito isso, mesmo que um produto $k$ já tenha sido exportado para o grupo "a", sua primeira aparição na pauta do grupo "b" será definida como "nova". Usando uma definição mais concreta: observa-se da base de dados deste estudo, que os produtos classificados no grupo HS4 igual a $6308^{11}$ só passaram a fazer parte da pauta exportadora no ano de 1992, no comércio entre Paraguai e Alemanha. Ou seja, esse produto não havia sido exportado nos anos 1989,1990 e 1991 e, por isso, é definido como um novo produto.

Para avaliar se a promulgação do Mercosul é capaz de criar mercado para novos produtos para terceiros países deve-se ter o cuidado de trabalhar somente com produtos que não faziam parte do fluxo de comércio bilateral antes da entrada em vigor do Mercosul (em $t-2$ e $t-1) .{ }^{12}$ Assim, o segundo filtro a ser aplicado na base trata-se da exclusão daqueles produtos para os quais já existia comércio antes da promulgação do Mercosul, nos anos de 1989 e 1990.

Contudo, após esse segundo filtro o regressando será composto tanto por produtos que começaram a ser comercializados após a instituição do Mercosul bem como por produtos que mesmo após a entrada em vigor desse acordo não foram incluídos na pauta de exportação. Sendo assim, o terceiro filtro aplicado à variável dependente foi a exclusão desses produtos que mesmo após a criação do Mercosul não passaram a ser exportados.

Assim, utilizando o modelo de escolha binária logit, o efeito do Mercosul sobre a probabilidade da criação de um novo mercado para o produto $k$ pode ser medido pela seguinte expressão:

$$
\begin{aligned}
& \operatorname{Pr}\left(\text { newp }_{i j k, t}\right)=\beta_{1} \operatorname{lgd} p_{i, t}+\beta_{2} \operatorname{lgd} p_{j, t}+\beta_{3} l x r_{i, t}+\beta_{4} \text { inside }_{i j k, t}+ \\
& +\beta_{5} \text { outside }_{i j k, t}+\beta_{6} \text { mercosur }_{i j, t}+\beta_{7} \text { spillover }_{i j k, t}+ \\
& +\beta_{8} \operatorname{lmodul} e_{i j, t}+\gamma_{t}+\epsilon_{i j k, t} ;
\end{aligned}
$$

em que $\operatorname{Pr}\left(n e w p_{i j k, t}\right)$ assume valor 1 se um novo produto $k$ for exportado pelo país $i$, membro do Mercosul, para um país $j$ fora do acordo comercial pela primeira vez no período $t ; \lg d p_{i, t}$ caracteriza-se como sendo o logaritmo do Produto Interno Bruto (PIB) do país de origem enquanto que $\lg d p_{j, t}$ o logaritmo do PIB do país de destino; já $l x r_{i, t}$ representa o logaritmo da taxa de câmbio real do país de origem; inside $e_{i j k, t}{ }^{13}$ caracteriza-se como sendo uma dummy que assume valor igual a um se o produto $k$ era exportado, antes da promulgação do Mercosul (no caso em análise os anos de 1989 e 1990), apenas para países membros do acordo e zero caso contrário, outside $e_{i j k, t}{ }^{13}$ é uma $d u m m y$ que assume um se o produto $k$ era comercializado apenas entre membros e não-membros em qualquer dos dois anos anteriores a promulgação do

\footnotetext{
${ }^{11}$ Compreendendo: têxteis, sortidos de tecidos e fios, com ou sem acessórios, para confecção de tapetes, tapeçarias, bordados, guardanapos e semelhantes, em embalagens para venda a retalho.

${ }^{12}$ Em que $t$ representa o ano da entrada em vigor do Mercosul, 1991.

${ }^{13}$ Note que um novo produto exportado para um terceiro país pode apresentar uma experiência anterior para um país membro ou para outro mercado não-membro ou para ambos, bem como não possuir nehuma experiência anterior.
} 
Mercosul, ou seja, nos anos de 1989 e 1990, e zero em caso contrário; por sua vez mercosur $r_{i j, t}$ representa uma dummy que assume um se o país exportador $i$ fazia parte do acordo de comércio e zero caso contrário; caracterizando o módulo da diferença entre os PIBs per capita do país de origem e de destino tem-se $\operatorname{lmodule}_{i j, t}$, sendo uma proxy para a similaridade entre os países com o intuito de capturar a proximidade de gostos e preferências dos consumidores. O coeficiente $\beta_{7}$ é de maior interesse já que representa o efeito do termo de interação entre inside $e_{i j k, t}$ e mercosur $r_{i j, t}{ }^{14}$ que determina a ocorrência do spillover do Mercosul. A variável $\gamma_{t}$ denota os efeitos fixos de tempo e $\epsilon_{i j k, t} \mathrm{o}$ termo de erro não-observável.

Salienta-se que a inclusão das variáveis $l g d p_{i, t}$ e $\lg d p_{j, t}$ no modelo econométrico tem como finalidade observar se acordos comerciais com países desenvolvidos possuem uma maior probabilidade de exportação para terceiros países. Quanto à inclusão do regressor $l x r_{i, t}$, justifica-se, uma vez que apreciações (depreciações) reais do câmbio poderiam diminuir (aumentar) a probabilidade de exportar um novo produto. Portanto, essa variável controla para qualquer mudança na competitividade dos exportadores. Por sua vez, as variáveis $i n s i d e_{i j k, t}$ e outside $e_{i j k, t}$ objetivam capturar, respectivamente, os efeitos de uma experiência anterior dentro e fora do Mercosul. Ou seja, se experiências anteriores possuem efeitos positivos sobre a probabilidade de se criar mercado para esses mesmos produtos, confirmando ou rejeitando a hipótese da existência de um mecanismo de aprendizagem - "learning by exporting" ou de uma economia de escala na exportação, respectivamente.

Para determinar a existência do efeito spillover do Mercosul, inclui-se a variável de interação spillover $_{i j k, t}$ na especificação econométrica. Importante salientar que se o parâmetro dessa variável reportar um sinal positivo, significa que a experiência anterior dos membros do Mercosul possui um efeito adicional para a criação de mercado para novos produtos exportados para os não-membros. A Tabela 1 apresenta as principais variáveis incluídas no modelo e uma síntese dos sinais esperados.

Destaca-se, ainda, que neste tipo de análise existe o risco da sobreposição de efeitos dos acordos comerciais assinados e, sendo assim, o intervalo de tempo entre cada "Regional Trade Agreement" é de fundamental importância para a identifição do efeito spillover "puro" do acordo em análise, i.e., independente de quaisquer efeitos de outros acordos. Nesse sentido, Molina (2010) destaca que adotar uma janela de setes anos (três anos antes, $t-3$, e três anos depois, $t+3)$, centrada em torno da data da entrada em vigor do Mercosul assim $t$, neste estudo, corresponde ao ano de 1991 - parece ser razoável para contornar o problema da sobreposição. Dessa forma, uma vez que o Mercosul foi o único acordo assinado por Argentina, Brasil, Paraguai e Uruguai dentro desse intervalo temporal (janela de sete anos), pode-se dizer que os resultados reportados por este artigo não apresentam o problema da sobreposição de efeitos.

Além disso, objetivando filtrar para outros efeitos, ${ }^{15}$ este artigo considera a criação de mercado para novos produtos para terceiros países como sendo apenas aqueles produtos que passaram a ser exportados nos cinco anos seguin-

\footnotetext{
${ }^{14}$ Essa interação dá origem à variável denominada de spillover $_{i j k, t}$.

${ }^{15}$ Conforme Molina (2010, p. 85), “os produtos exportados para não-membros em $t+6$ ou mais períodos não podem ser observados, mas aumentar o lapso temporal poderá capturar outros efeitos de demanda ou oferta".
} 
Tabela 1: Descrição das variáveis independentes

\begin{tabular}{|c|c|c|}
\hline Variáveis & $\begin{array}{c}\text { Sinal } \\
\text { esperado }\end{array}$ & Efeito sobre a variável dependente \\
\hline $\lg d p_{i, t}$ & $>0$ & Impacto do PIB do país exportador \\
\hline $\lg d p_{j, t}$ & $>0$ & Impacto do PIB do país importador \\
\hline$l x r_{i, t}$ & $<0$ & $\begin{array}{l}\text { Controlar mudanças de competitividade } \\
\text { do exportador }\end{array}$ \\
\hline inside $_{i j k, t}$ & $>0$ & $\begin{array}{l}\text { Indica se a experiência de exportação pré- } \\
\text { via para países membros afeta a exportação } \\
\text { de novos produtos }\end{array}$ \\
\hline outside $e_{i j k, t}$ & $>0$ & $\begin{array}{l}\text { Controlar o efeito de haver exportação para } \\
\text { um país não-membro antes do acordo co- } \\
\text { mercial }\end{array}$ \\
\hline$m e r c o s u r_{i j, t}$ & $>0$ & $\begin{array}{l}\text { Denota a influência de fazer parte do } \\
\text { acordo sobre as exportações para países } \\
\text { não membros }\end{array}$ \\
\hline spillover $_{i j k, t}$ & $>0$ & $\begin{array}{l}\text { Indica a existência do spillover devido ao } \\
\text { acordo comercial }\end{array}$ \\
\hline $\operatorname{lmodule}_{i j, t}$ & $<0$ & $\begin{array}{l}\text { Expressa o efeito da similaridade de renda } \\
\text { dos países do fluxo de comércio }\end{array}$ \\
\hline
\end{tabular}

tes à promulgação do Mercosul $(t+5) \cdot{ }^{16}$ Contudo, como um teste de robustez, este artigo adotou um intervalo temporal maior entre a promulgação do Mercosul e a exportação de novos produtos para países não-membros, ou seja, considerou como novos produtos exportados para terceiros países aqueles que passaram a ser exportados ao longo de todo o período amostral após a implementação do Mercosul $(t+19)$. Sendo assim, este artigo estimou dois modelos distintos em que, primeiramente, analisou o efeito spillover do Mercosul considerando apenas a criação de mercado para novos produtos durante os cincos anos posteriores $(t+5)$ à implementação do Mercosul, definido como Modelo Restrito, e, posteriormente, realizou-se uma outra estimação considerando a criação de mercado para novos produtos ao longo de todo o período após a constituição do Mercosul $(t+19)$, denominado de Modelo Irrestrito.

Por fim, torna-se fundamental destacar uma importante limitação associada à estimação do Modelo (5) via logit (e/ou probit), qual seja: modelos de dados em painel não-lineares com efeitos fixos podem gerar estimativas viciadas devido ao problema de "parâmetros incidentais", i.e., a teoria assintótica desses modelos demonstra que o número de "nuisance parameters" cresce com a amostra. ${ }^{17}$ Diante disso, e visando controlar por fatores que são particulares ao par de países, $i j$, porém invariantes no tempo, optou-se pela estimação do Modelo 6 por meio de um modelo de probabilidade linear (MPL):

\footnotetext{
${ }^{16}$ O que corresponde aos anos de 1992,1993,1994,1995 e 1996.

${ }^{17}$ Esse problema foi estabelecido no estudo de Neyman \& Scott (1948). Para uma discussão recente, ver Fernández-Val \& Weidner (2016).
} 


$$
\begin{array}{r}
\operatorname{Pr}\left(\text { newp } p_{i j k, t}\right)=\beta_{1} \operatorname{lgdp}_{i, t}+\beta_{2} l g d p_{j, t}+\beta_{3} \operatorname{lxr}_{i, t}+\beta_{4} \text { inside }_{i j k, t}+ \\
+\beta_{5} \text { outside }_{i j k, t}+\beta_{6} \text { mercosur }_{i j, t}+\beta_{7} \text { spillover }_{i j k, t}+ \\
+\beta_{8} \text { lmodule }_{i j, t}+\gamma_{t}+\eta_{i j}+\epsilon_{i j k, t}
\end{array}
$$

em que $\eta_{i j}$ denota os efeitos fixos por pares de países. Sabe-se que o MPL possui uma limitação associada à possibilidade de gerar valores ajustados para a variável dependente fora do intervalo $(0,1)$. Logo, esse modelo será incluído como um teste de robustez, uma vez que ele controla por fatores nãoobservados associados ao par $i j$ e invariantes no tempo.

\section{Resultados e Discussões}

A Tabela 2 sintetiza os principais resultados do estudo. Na primeira coluna de cada modelo, Restrito e Irrestrito, apresentam-se os resultados para os parâmetros e seus desvios padrões. A segunda coluna destina-se a expor os efeitos marginais. De uma forma geral, não há diferença expressiva entre os dois modelos apresentados. ${ }^{18}$ Contudo, essa similaridade é justificável, dado que o Mercosul estabelece um compromisso entre os Estados signatários que os proíbe de negociarem acordos bilaterais de livre-comércio de forma independente, sem os outros membros do bloco. Em outras palavras, estabelece o compromisso dos membros do Mercosul de negociarem de forma conjunta os acordos comerciais que incluem preferências tarifárias com terceiros países. Sendo assim, e ciente de que a diferença principal entre os modelos restrito e irrestrito se dá no intervalo temporal considerado para observar um novo produto, é natural que os resultados sejam próximos.

Via de regra, todos os coeficientes estimados apresentaram os sinais esperados e significância estatística. Os sinais positivos para $l g d p_{i, t}$ e $l g d p_{j, t}$, Tabela 2, sugerem que países participantes de acordos com economias desenvolvidas possuem uma maior probabilidade de exportarem para terceiros países. Já para a variável $l x r_{i, t}$ o sinal negativo implica que quanto menos apreciado estiver o câmbio maior a probalidade de se exportar para um país fora da área do Mercosul. As experiências anteriores dentro da área do Mercosul, inside $e_{i j k, t}$, reportaram um sinal positivo, porém, não significativo. Já a variável outside $e_{i j k, t}$, foi positiva e estatisticamente diferente de zero, demonstrando que experiências anteriores fora da área do Mercosul possuem um efeito positivo sobre a probabilidade de exportação desse mesmo produto para não-membros. Esse último resultado ratifica a hipótese da existência de uma economia de escala na exportação.

Focando no efeito spillover, ambos os modelos reportam coeficientes positivos e significativos. Nota-se ainda que, quanto maior a similaridade entre as duas economias $\left(\operatorname{lmodul}_{i j, t}\right)$, maior será a probabilidade de criação de mercado para novos produtos para terceiros países. Dito isso, nota-se que, após controlar para outros fatores que possam impactar sobre a probabilidade da criação de mercado para novos produtos para não-membros, a variável de interesse spillover $_{i j k, t}$ possui um efeito positivo e estatisticamente significante sobre essa probabilidade, sendo que experiências anteriores de exportação para

\footnotetext{
${ }^{18}$ Por brevidade não foram reportados na Tabela 2 os valores para os coeficientes dos efeitos fixos ano.
} 
Tabela 2: Probabiliade de criação de novos mercados para nãomembros

\begin{tabular}{|c|c|c|c|c|}
\hline \multirow{2}{*}{ Variável } & \multicolumn{2}{|c|}{ Modelo Restrito } & \multicolumn{2}{|c|}{ Modelo Irrestrito } \\
\hline & Coeficiente & Efeito Marginal & Coeficiente & Efeito Marginal \\
\hline $\operatorname{lgd} p_{i, t}$ & $0,0950^{a}(0,0062)$ & $0,0097^{a}(0,0006)$ & $0,0955^{a}(0,0061)$ & $0,0098^{a}(0,0006)$ \\
\hline $\lg d p_{j, t}$ & $0,0793^{a}(0,0031)$ & $0,0081^{a}(0,0003)$ & $0,0788^{a}(0,0030)$ & $0,0081^{a}(0,0003)$ \\
\hline$l x r_{i, t}$ & $-0,0259^{a}(0,0025)$ & $-0,0027^{a}(0,0030)$ & $-0,0257^{a}(0,0025)$ & $-0,0026^{a}(0,0003)$ \\
\hline inside $_{i j k, t}$ & $0,0022 \quad(0,0146)$ & $0,0002 \quad(0,0015)$ & $0,0101 \quad(0,0144)$ & $0,0010 \quad(0,0015)$ \\
\hline outside $i j k, t$ & $0,3481^{a}(0,0257)$ & $0,0318^{a}(0,0021)$ & $0,3329^{a}(0,0254)$ & $0,0306^{a}(0,0021)$ \\
\hline mercosur $_{i j, t}$ & $0,8029^{a}(0,0218)$ & $0,1002^{a}(0,0032)$ & $0,7703^{a}(0,0215)$ & $0,0955^{a}(0,0031)$ \\
\hline spillover $_{i j k, t}$ & $0,1757^{a}(0,0261)$ & $0,0190^{a}(0,0030)$ & $0,1847^{a}(0,0256)$ & $0,0204^{a}(0,0029)$ \\
\hline $\operatorname{lmodule}_{i j, t}$ & $-0,0620^{a}(0,0047)$ & $-0,0064^{a}(0,0005)$ & $-0,0621^{a}(0,0046)$ & $-0,0064^{a}(0,0005)$ \\
\hline Amostra & \multicolumn{2}{|c|}{328.140} & \multicolumn{2}{|c|}{338.822} \\
\hline
\end{tabular}

Notas: Erros padrões entre parênteses. Significância estatística: ${ }^{a} 1 \%,{ }^{b}{ }^{2} \mathrm{e}^{c} 10 \%$.

o produto $k$ dentro da área do Mercosul implicaria em uma probabilidade de 1,9\% desse mesmo produto ser exportado para países fora da área desse bloco.

Embora não configure um impacto expressivo, pode-se entender esse resultado como um efeito ignorado pela literatura pregressa. De uma forma geral, os estudos concentram-se no impacto direto do Mercosul sobre as exportações. A variável mercosur $r_{i j, t}$, por exemplo, mensura o efeito do bloco sobre a margem extensiva (novos produtos) - em média 10\%. Esse resultado é similar ao reportado por Figueiredo \& Loures (2016). Contudo, também devese considerar os efeitos sobre o grupo de países não-tratados. Nesse sentido, pode-se dizer que o Mercosul está associado a uma probabilidade de aproximadamente $12 \%$ de criação de margem extensiva de comércio.

Convém ressaltar que, embora divirjam na magnitude, os resultados listados na Tabela 2 corroboram os achados da literatura em relação aos efeitos dos RTAs sobre a margem extensiva do comércio. O estudo Molina (2010), por exemplo, encontra um efeito spillover das RTAs muito próximo aos aqui reportados. Com um foco específico no Mercosul, Nicita et al. (2003) sugerem que o acordo serve como uma plataforma de acesso para terceiros mercados das exportações do Paraguai. O efeito dos RTAs nas exportações para terceiros países também é demonstrado em Borchert (2007), que encontra uma expansão dos produtos mexicanos para países fora do NAFTA, especificamente para a América Latina e Central.

\subsection{Teste de Robustez}

Como mencionado na Subseção 2.3, as estimativas logit (probit) não são robustas à inclusão de efeitos fixos. Nesse sentido, a estimação do Modelo (5) requer a consideração de estruturas lineares, tal como o MPL. Sendo assim, será possível incluir os efeitos fixos considerados pela literatura. A Tabela 3 apresenta três especificações: i) coluna 2, com o modelo com efeito fixo por ano; ii) coluna 3 , com efeitos por pares de países e; iii) coluna 4, com efeitos fixos de ano e pares de países. Regra geral, as magnitudes dos resultados não se alteram de forma expressiva quando comparadas com as estimativas logit apresentadas na seção anterior. Observam-se a manutenção dos sinais esperados, com duas exceções: os sinais para $l x r_{i, t}$ e $l \bmod u l e_{i j, t}$ no modelo $\mathrm{MPL}^{2}$ que reportaram sinais positivos e significativos.

Assim como para a especificação baseline do artigo, Modelo (5), os regressores $\lg d p_{i, t}$ e $\lg d p_{j, t}$ reportaram sinais positivos e significância estatística para 
Tabela 3: Resultados para os Modelos de Probabilidade Linear (MPL)

\begin{tabular}{|c|c|c|c|}
\hline Variável & $\overline{\text { MPL }^{1}}$ & $\mathrm{MPL}^{2}$ & $\mathrm{MPL}^{3}$ \\
\hline $\lg d p_{i+t}$ & $0,0114^{a}(0,0007)$ & $0,2320^{a}(0,0054)$ & $0,0520^{a}(0,0111)$ \\
\hline $\lg d p_{i, t}$ & $0,0080^{a}(0,0003)$ & $0,1040^{a}(0,0041)$ & $0,0237^{a}(0,0047)$ \\
\hline$l x r_{i, t}$ & $-0,0018^{a}(0,0002)$ & $0,0072^{a}(0,0005)$ & $0,0008 \quad(0,0007)$ \\
\hline inside $_{i j k, t}$ & $0,0015 \quad(0,0014)$ & $0,0011 \quad(0,0015)$ & $0,0013 \quad(0,0014)$ \\
\hline outside $i j k, t$ & $0,0380^{a}(0,0026)$ & $0,0373^{a}(0,0030)$ & $0,0373^{a}(0,0030)$ \\
\hline $\operatorname{mercosur}_{i j, t}$ & $0,1040^{a}(0,0026)$ & $0,0902^{a}(0,0052)$ & $0,0963^{a}(0,0055)$ \\
\hline spillover $_{i j k, t}$ & $0,0240^{a}(0,0032)$ & $0,0431^{a}(0,0034)$ & $0,0418^{a}(0,0034)$ \\
\hline $\operatorname{lmodule} i j, t$ & $-0,0075^{a}(0,0005)$ & $0,0004^{a}(0,0013)$ & $0,0008 \quad(0,0013)$ \\
\hline Amostra & \multicolumn{3}{|c|}{338.822} \\
\hline Efeito fix & Sim & Não & Sim \\
\hline Efeito fixo par & Não & Sim & Sim \\
\hline
\end{tabular}

Notas: Erros padrões entre parênteses. Significância estatística: ${ }^{a} 1 \%,{ }^{b}{ }^{b} \% \mathrm{e}^{c} 10 \%$.

os três modelos de robustez ${ }^{19}$, sinalizando a propensão de acordos com economias desenvolvidas de exportarem para terceiros países. Por sua vez, para a variável $l x r_{i, t}$, os sinais foram negativo no modelo $\mathrm{MPL}^{1}$ e, ao contrário do modelo baseline, positivo nos modelos $\mathrm{MPL}^{2}$ e $\mathrm{MPL}^{3}$, porém significativo apenas naquele. Já para as variáveis explicativas inside $_{i j k, t}$ e outside $e_{i j k, t}$ os sinais reportados nos três modelos foram positivos, porém, com significância estatística somente para outside $e_{i j k, t}$. Sendo assim, experiências anteriores à promulgação do Mercosul, entre países membros e não-membros desse RTA, impactam positivamente sobre a probabilidade de exportar o mesmo produto para não-membros, mesmo resultado obtido no Modelo (5).

Quanto à variável de controle para RTA, mercosur $_{i j, t}$, essa reportou o sinal esperado, além de ser estatisticamente significante e, sendo assim, indicando que participar do acordo aumenta a probabilidade de criação de mercado para novos produtos para terceiros países. Para a variável de interesse, spillover $_{i j k, t}$, os sinais reportados nos três modelos ratificam os resultados do modelo baseline, evidenciando que experiências anteriores no comércio bilateral para o produto $k$ entre os Estados membros do Mercosul resultaria em uma maior probabilidade de exportar o mesmo produto para países não-membros. A similaridade entre as duas economias, $l \bmod u l e_{i j, t}$, assim como no modelo baseline, apresentou sinal negativo no modelo $\mathrm{MPL}^{1}$, significando que, quanto maior a similaridade entre os países, maior a probabilidade da criação de comércio para terceiros países. Porém, os modelos $\mathrm{MPL}^{2}$ e $\mathrm{MPL}^{3}$ reportaram sinal positivo, sendo significativo apenas no $\mathrm{MPL}^{2}$.

\section{Considerações finais}

Este artigo se concentrou em uma área ainda pouco explorada pela literatura empírica de economia internacional, qual seja, a captação dos efeitos indiretos dos Acordos Regionais de Comércio (Regional Trade Agreements). Para tanto, definiu-se efeito spillover como a capacidade de criação de mercado para novos produtos fora do bloco comercial. Concentrando-se no Mercosul, observou-se que o seu efeito direto - criação de mercado para novos produtos dentro do bloco - situa-se próximo de $10 \%$. Adicionalmente, seu efeito spillover foi de

${ }^{19} \mathrm{MPL}^{1}, \mathrm{MPL}^{2}$ e $\mathrm{MPL}^{3}$. 
cerca de 2\%. A captação desse efeito adicional constitui a principal contribuição deste artigo, abrindo espaço para investigacões futuras voltadas para mensurações mais precisas envolvendo o efeito do Mercosul e indicadores de crescimento e bem-estar dos países membros.

Por fim, e seguindo as orientações contidas em Preusse (2001), ressalta-se que os benefícios do efeito spillover do Mercosul podem ser potencializados a partir de uma maior coordenação das políticas adotadas entre os países membros de forma a estabilizar o sistema de incentivos para investidores intrabloco. Além disso, o autor recomenda uma maior flexibilização da obrigatoriedade dos países membros adotarem um política comercial comum contra não-membros, o que estimularia o comércio com terceiros países. Ademais, assim como em Bean (1992) e Galvão Jr (2008), propõe-se a resolução de conflitos das políticas comerciais nos setores mais sensíveis do bloco.

\section{Referências Bibliográficas}

Azevedo, A. F. Z. (2008), 'Mercosul: o impacto da liberalização preferencial e as perspectivas para a união aduaneira'. in Baldwin, R. E., Di Nino, V., Fontagne, L., De Santis, R. A. \& Taglioni, D., eds, Study on the Impact of the Euro on Trade and Foreign Direct Investment, Brussels: European Commission. (Economic Papers 321).

Baldwin, R. \& Di Nino, V. (2008), The newly-traded goods hypothesis: evidence from the trade data, in R. E. Baldwin, V. Di Nino, L. Fontagne, R. A. De Santis \& D. Taglioni, eds, 'Study on the Impact of the Euro on Trade and Foreign Direct Investment', SSRN Electronic Journal.

Bean, C. R. (1992), 'Economic and monetary union in Europe', Journal of Economic Perspectives 6(4), 31-52.

Blyde, J. S., Fernández-Arias, E. \& Giordano, P. (2008), Deepening integration in Mercosur: Dealing with Disparities, Washington, DC: Inter-American Development Bank.

Borchert, I. (2007), 'Preferential Trade Liberalization and the PathDependent Expansion of Exports'. St. Gallen: University of St. Gallen, Department of Economics. (Discussions Paper No. 2007-06).

Cordeiro, B. F. \& Rodrigues Jr, M. (2016), Os impactos do Mercosul sobre o comércio: uma abordagem gravitacional, Master's thesis. São Paulo: FEA-USP. Dissertação de Mestrado).

Fernández-Val, I. \& Weidner, M. (2016), 'Individual and time effects in nonlinear panel data models with large N', Journal of Econometrics 196, 291-312.

Figueiredo, E. \& Loures, A. (2016), 'O Efeito do Mercosul Sobre a Comercialização de Novos Produtos', Revista Brasileira de Economia 70(3), 305-314.

Galvão Jr, R. F. (2008), Regional Competitiveness Policies for Deeper Integration in Mercosur, in Deepening integration in Mercosur: Dealing with Disparities, Washington, DC: Inter-American Development Bank.

Krugman, P. (1980), 'Scale economies, product differentiation, and the pattern of trade', The American Economic Review 70(5), 950-959. 
Loures, A. \& Figueiredo, E. (2017), 'Uma nota sobre o impacto do comércio internacional no crescimento de economias em desenvolvimento', Revista Brasileira de Economia 71(4), 453-461.

Molina, A. C. (2010), 'Are preferential agreements stepping stones to other markets?'. Geneva: The Graduate Institute of International and Development Studies. (IHEID Working Papers 13-2010).

Moore, F. T. (1959), 'Economies of scale: some statistical evidence', The Quarterly Journal of Economics 73(2), 232-245.

Neyman, J. \& Scott, E. L. (1948), 'Consistent estimates based on partially consistent observations', Econometrica 16(1), 1-32.

Nicita, A., Olarreaga, M. \& Soloaga, I. (2003), 'The Region as an Export Platform to the World?: The Case of Mercosur', Cuadernos de economia 40(121), 442-451.

Olarreaga, M. \& Soloaga, I. (1998), 'Endogenous tariff formation: the case of Mercosur', The World Bank Economic Review 12(2), 297-320.

Preusse, H. G. (2001), 'Mercosur: another failed move towards regional integration?', The World Economy 24, 911-931.

Reis, M., Azevedo, A. F. Z. \& Lélis, M. T. C. (2014), 'Os efeitos do novo regionalismo sobre o comércio', Estudos Econômicos 44(2), 351-381.

Santos Silva, J. M. C., Tenreyro, S. \& Wei, K. (2014), 'Estimating the extensive margin of trade', Journal of International Economics 93(1), 67-75.

Yeats, A. J. (1997), Does Mercosur's trade performance raise concerns about the effects of regional trade arrangements?, Technical report. Washington, DC: World Bank. (Policy Research Working Paper Series 1729). 


\section{Apêndice A-1}

Tabela 4: Lista dos países de destino

\begin{tabular}{|c|c|c|c|}
\hline África do Sul & Croácia & Islândia & Quênia \\
\hline Albânia & Cuba & Israel & Reino Unido \\
\hline Alemanha & Dijibuti & Itália & Rep. Centro-Africana \\
\hline Andorra & Dinamarca & Jamaica & Rep. Checa \\
\hline Angola & Dominica & Japão & Rep. Dominicana \\
\hline Antígua e Barbuda & Egito & Jordânia & Rep. Quirguiz \\
\hline Antilhas Holandesas & El Salvador & Kuwait & Ruanda \\
\hline Arábia Saudita & Emirados Árabes Reunidos & Laos & Rússia \\
\hline Argélia & Equador & Letônia & Samoa \\
\hline Argentina* & Eritreia & Líbano & Santa Helena \\
\hline Armênia & Eslováquia, Rep. & Libéria & Santa Lúcia \\
\hline Aruba & Eslovênia & Líbia & São Cristóvão e Nevis \\
\hline Austrália & Espanha & Lituânia & São Tomé e Príncipe \\
\hline Áustria & Estados Unidos & Macau & São Vicente e Granadinas \\
\hline Azerbaijão & Estônia & Macedônia, FYR & Seicheles \\
\hline Bahamas & Etiópia & Madagascar & Senegal \\
\hline Bangladesh & Fiji & Malásia & Serra Leoa \\
\hline Barbados & Filipinas & Malaui & Singapura \\
\hline Barém & Finlândia & Maldivas & Síria \\
\hline Belize & França & Mali & Sri Lanka \\
\hline Benin & Gabão & Malta & Sudão \\
\hline Bermudas & Gâmbia & Marrocos & Suécia \\
\hline Bielorrússia & Gana & Maurício & Suíça \\
\hline Bolívia & Geórgia & Mauritânia & Suriname \\
\hline Bósnia e Herzegovina & Gibraltar & México & Tailândia \\
\hline Brasil $^{*}$ & Granada & Moçambique & Taiwan \\
\hline Brunei Darussalam & Grécia & Moldávia & Tajiquistão \\
\hline Bulgária & Guatemala & Mongólia & Tanzânia \\
\hline Burquina Faso & Guiana & Montserrat & Togo \\
\hline Burúndi & Guiné & Myanmar & Tonga \\
\hline Butão & Guiné-Bissau & Nepal & Trinidad e Tobago \\
\hline Cabo Verde & Guiné Equatorial & Nicarágua & Tunísia \\
\hline Camarões & Haiti & Níger & Turquemenistão \\
\hline Camboja & Honduras & Nigéria & Turquia \\
\hline Canadá & Hong Kong & Noruega & Ucrânia \\
\hline Catar & Hungria & Nova Caledônia & Uganda \\
\hline Cazaquistão & Iêmen & Nova Zelândia & Uruguai* \\
\hline Chile & Ilhas Cayman & Omã & Vanuatu \\
\hline China & Ilhas Malvinas & Países Baixos & Venezuela \\
\hline Chipre & Ilhas Marianas do Norte & Panamá & Vietnã \\
\hline Colômbia & Ilhas Marshall & Papua-Nova Guiné & Zâmbia \\
\hline Comores & Ilhas Virgens Britânicas & Paquistão & Zimbábue \\
\hline Congo, Rep. Dem. do & Índia & Paraguai* & \\
\hline Coreia, Rep. da & Indonésia & Peru & \\
\hline Coreia do Norte & Irã, Rep. Islâmica do & Polinésia Francesa & \\
\hline Costa do Marfim & Iraque & Polônia & \\
\hline Costa Rica & Irlanda & Portugal & \\
\hline
\end{tabular}

Nota: O símbolo de asterisco indica os países de origem, $i$, mas que também são destino, $j$. 